\title{
Product News
}

\section{Atomic Emission Spectrometer Offers Stability, Accuracy, Precision}

Leco introduces the GDS900 glow discharge atomic emission spectrometer, which is designed specifically for routine bulk elemental determination in most conductive solid metal matrices. It features improved performance, stability, accuracy, and precision in steel, iron (including as-cast), aluminum, copper, zinc, nickel, cobalt, tungsten, and titanium. Low-melting alloys, resulfurized steel, powder metals, and other historically difficult-to-analyze materials can be analyzed in a production environment. It is a versatile solution in that sample types include bulk, sheet, wire, pressed, sectioned, and mounted samples, and various sample holders are available to meet nonstandard sample forms.

Advantages include simple, linear calibrations due to a controlled excitation that is done away from the surface, and the choice of 4 and $2 \mathrm{~mm}$ anodes to optimize spot size. The inherent sensitivity, dynamic range, and linearity of CCD detectors coupled with the GD source ensure stability, flexibility, and performance for all bulk elemental applications with full wavelength coverage from 160 to $460 \mathrm{~nm}$. Automatic source cleaning between samples saves time and minimizes matrix effects for increased precision. The GDS900 also introduces Leco's Cornerstone brand software

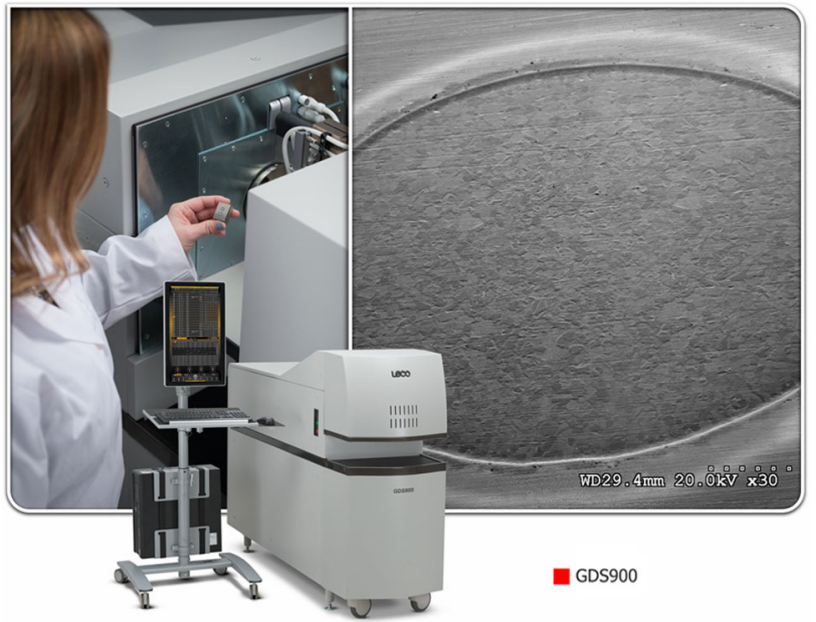

Leco's GDS900 glow discharge atomic emission spectrometer

and a touch-screen interface, for increased usability, simplified reporting, and streamlined analysis times.

The GDS900 is available in several model configurations, so users can choose the model that best fits the application, including a nitrogen purge recirculation option.

For more information: LECO Corporation, 3000 Lakeview Ave., Saint Joseph, MI 49085; tel: 800/292-6141 or 269/9855496; fax: 269/982-8977; e-mail: sales@leco.com; web: www.leco.com. 